\title{
Voltage-Dependent L-Type Calcium Channel Subunit Alpha-1S
}

National Cancer Institute

\section{Source}

National Cancer Institute. Voltage-Dependent L-Type Calcium Channel Subunit Alpha-1S. NCI Thesaurus. Code C150198.

Voltage-dependent L-type calcium channel subunit alpha-1S (1873 aa, 212 kDa) is encoded by the human CACNA1S gene. This protein plays a role in voltage-dependent calcium transport in muscle cells. 